A. Neff ${ }^{1}$ - G. Mühlberger ${ }^{1} \cdot$ M. Karoglan ${ }^{1} \cdot$ A. Kolk ${ }^{1} \cdot$ S. Koch ${ }^{1} \cdot$ W. Mittelmeier ${ }^{2}$

H.Schieferstein ${ }^{1,2} \cdot$ D. Scheruhn ${ }^{2} \cdot$ H.-H. Horch ${ }^{1}$

${ }^{1}$ Klinik und Poliklinik für Mund-, Kiefer-, Gesichtschirurgie der Technischen Universität,

Klinikum rechts der Isar, München

${ }^{2}$ Klinik für Orthopädie und Sportorthopädie der Technischen Universität,

Klinikum rechts der Isar mit Abteilung Biomechanik an der ZHS München

\title{
Stabilitåt der Osteosynthese bei Gelenkwalzenfrakturen in Klinik und biomechanischer Simulation
}

\section{Mund Kiefer GesichtsChir (2004) 8:63-74}

Fälschlich wurden S. Koch und H. Schieferstein nicht als Autoren genannt.

Die elektronische Version des Originalartikels finden Sie unter http://dx.doi.org/10.1007/ s10006-004-0529-9

Priv.-Doz.Dr.Dr.A.Neff

Klinik und Poliklinik für Mund-, Kiefer-, Gesichtschirurgie der Technischen Universität, Klinikum rechts der Isar, Ismaninger Straße 22, 81675 München Tel.: 089-41402926, Fax: 089-44718241 E-mail: A.Neff@Irz.tu-muenchen.de 